\title{
Regularity for Quasi-linear Elliptic Systems with Discontinuous Coefficients
}

\author{
S. Zheng and Z. Feng
}

Communicated by Y. Charles Li, received October 10, $200 \%$.

\begin{abstract}
In this paper we study regularity and partial regularity for the weak solution of a class of general quasi-linear elliptic equations and systems, which are of the quasi-linear main coefficients satisfying the VMO conditions in $x$ uniformly with respect to $u$, and of the lower order items satisfying controllable growth.
\end{abstract}

\section{Contents}

1. Introduction $\quad 87$

2. Preliminaries 90

3. Proofs of Main Results $\quad 92$

4. Conclusion 97

Acknowledgments $\quad 98$

$\begin{array}{ll}\text { References } & 98\end{array}$

\section{Introduction}

In this paper, our purpose is to study regularity and partial regularity of weak solutions for the general quasi-linear elliptic equation and system of divergence form:

$$
-D_{\alpha}\left[A_{i j}^{\alpha \beta}(x, u) D_{\beta} u^{j}+a_{i}^{\alpha}(x, u)\right]=b_{i}(x, u, \nabla u), \quad \text { in } \Omega,
$$

1991 Mathematics Subject Classification. 35J60, 35B45.

Key words and phrases. VMO function, controllable growth, reserve Hölder inequality, partial regularity, weak solution.

Main contents of this paper have been presented at the Third International Symposium on Nonlinear Sciences and Applications, Fudan University, Shanghai, China, June 6-10, 2007 (Conference web: http://www.sss-nonlinear.org/webs/pc.htm). The work is supported by NSF(China) Grant 10671022 and NSF(USA) Grant CCF-0514768. 
for $\alpha, \beta=1,2, \cdots, n ; i, j=1,2, \cdots, N$, which contains the lower order items $b_{i}(x, u, \nabla u)$ satisfying controllable growth, where $\Omega$ is a domain of the Euclidean space $\mathbf{R}^{n}(n \geq 2)$, and $u: \Omega \rightarrow \mathbf{R}^{N}$ is a vector-valued function and $u \in W_{l o c}^{1,2}\left(\Omega, \mathbf{R}^{N}\right)$ is any weak solution of system (1.1) in the distributional sense:

$$
\int_{\Omega} A_{i j}^{\alpha \beta}(x, u) D_{\beta} u^{j} D_{\alpha} \phi^{i} d x=\int_{\Omega}\left[-a_{i}^{\alpha}(x, u) D_{\alpha} \phi^{i}+b_{i}(x, u, \nabla u) \phi^{i}\right] d x,
$$

for any $\phi \in C_{0}^{\infty}\left(\Omega, \mathbf{R}^{N}\right)$.

If $A_{i j}^{\alpha \beta}(x, u) \in C\left(\Omega \times \mathbf{R}^{N}\right)$, when $N=1$, an interior Hölder estimate can be obtained by using the classical Schauder approach and an $L^{p}$ estimate can be obtained by using the potential theory $[1,2]$. When $N>1$, Giaquinta and Modica $[3,4]$ investigated partial regularity of weak solutions of system (1.1) in the Morrey space $L^{2, \lambda}$ and the Campanato space $\mathcal{L}^{2, \lambda}[5,6]$. For linear divergence equations with bounded measurable coefficients, De Giorgi [7] and Nash [8] first established an interior Hölder estimate in the scalar case. Afterward, many researchers had obtained analogous results for general nonlinear single equation essentially by applying the technique of De Giorgi-Nash-Moser's iteration [1, 2, 9,10 etc.], and regularity results for some second-order elliptic partial differential equations related to the primitive equations were derived by $\mathrm{Hu}$ and his co-workers $[11,12]$. But a sharp Hölder exponent was not shown by way of the iteration technique and the Harnack inequality. So in this paper we are interested in establishing a sharp regularity of weak solutions reflected by the integrability of $f_{i}^{\alpha}(x)$ and $g_{i}(x)$ under certain minimal assumptions on the quasi-linear coefficient operators $A_{i j}^{\alpha \beta}(x, u)$, in particular, which possibly have discontinuous coefficients. Partial regularity of weak solutions to nonlinear elliptic systems satisfying a Dini condition was studied by Wolf [13]. The VMO (vanishing mean oscillation) function, introduced by Sarason [14], is of a number of good properties, which is not shared by general bounded measurable functions and BMO (bounded mean oscillation) functions. Another motivation for studying this problem comes from the recent development of the $L^{p}$-Schauder theory for linear and nonlinear elliptic and parabolic equations with $V M O \bigcap L^{\infty}$ coefficients by means of the harmonic analysis [15-21], and local minimizers of functionals by way of the freezing coefficient method [22]. So a naturally generalized assumption in this paper is to suppose that coefficients $A_{i j}^{\alpha \beta}$ are of vanishing mean oscillation in $x \in \Omega$ uniformly with respect to $u \in \mathbf{R}^{N}$, this is because we allow the coefficient VMO to be dependent on the variable $x$, and to be continuous with respect to $u$. However, the assumption of continuity with respect to $u$ is necessary and can not be removed by any standard argument in the literature. The main difficulty lies in that the composition of the VMO function with the $C^{\infty}$ function is not a priori VMO.

Here let us recall the definition of the VMO function and state some basic conditions which we will need to use in our main results.

Definition 1. [1, 23] A locally integrable function $f$ is said to belong to $B M O(\Omega)$ (the space of bounded mean oscillation in $\Omega$ ), if $f \in L_{l o c}(\Omega)$ and for any $0<a<\infty$, we have

$$
M_{a}(f, \Omega)=\sup _{x \in \Omega, 0<\rho<a}|\Omega(x, \rho)|^{-1} \int_{\Omega(x, \rho)}\left|f(y)-f_{x, \rho}\right| d y<+\infty
$$


where $\Omega(x, \rho)=\Omega \cap B(x, \rho)$ with any open ball $B(x, \rho)$ in $\mathbf{R}^{n}$ centered at $x$ of radius $\rho$, and $f_{x, \rho}:=f_{\Omega(x, \rho)} f(y) d y=\frac{1}{|\Omega(x, \rho)|} \int_{\Omega(x, \rho)} f(y) d y$.

Definition 2. [13] A function $f \in L_{l o c}(\Omega)$ is said to be in $V M O(\Omega)$ (the space of vanishing mean oscillation in $\Omega$ ), if

$$
M_{0}(f)=\lim _{a \rightarrow 0} M_{a}(f, \Omega)=0 .
$$

Suppose that $f \in B M O(\Omega)$, then it actually satisfies a stronger version of following property: for any $1<p<+\infty$, it follows that $f$ locally belongs to $L^{p}(\Omega)$. Moreover, we have [23]

$$
\left(|\Omega(x, \rho)|^{-1} \int_{\Omega(x, \rho)}\left|f(y)-f_{x, \rho}\right|^{p} d y\right)^{\frac{1}{p}} \leq C(p) M_{a}(f, \Omega),
$$

for any $0<\rho<a$. For system (1.1), we suppose that coefficient operators $A_{i j}^{\alpha \beta}(x, u)$, and the lower order items $a_{i}^{\alpha}(x, u)$ and $b_{i}(x, u, \nabla u)$ satisfy the following four conditions:

(H1) (Ellipticity). There exists a constant $\nu>0$ such that

$$
A_{i j}^{\alpha \beta}(x, u) \xi_{\alpha}^{i} \xi_{\beta}^{j} \geq \nu|\xi|^{2}, \quad \forall x \in \Omega, \forall u \in W_{l o c}^{1,2}, \forall \xi \in \mathbf{R}^{n N} .
$$

(H2) $\left(V M O \cap L^{\infty}\right.$ Property). Assume that $A_{i j}^{\alpha \beta}\left(x, u_{0}\right)$ belongs to $V M O \cap L^{\infty}(\Omega)$ uniformly with respect to $u_{0} \in \mathbf{R}^{N}$. That is,

$$
\lim _{a \rightarrow 0} M_{a}\left(A_{i j}^{\alpha \beta}\left(\cdot, u_{0}\right)\right)=0
$$

uniformly with respect to $u_{0} \in \mathbf{R}^{N}$, and there exists a positive constant $L$ such that $\left|A_{i j}^{\alpha \beta}(x, u)\right| \leq L$ for all $x \in \Omega$ and $u \in \mathbf{R}^{N}$.

(H3) (Continuity). There exist a constant $C$ and a continuous concave func$\operatorname{tion}^{1} \omega: \mathbf{R}^{+} \rightarrow \mathbf{R}^{+}$with $\omega(0)=0,0 \leq \omega \leq 1$ such that

$$
\left|A_{i j}^{\alpha \beta}\left(x_{0}, u\right)-A_{i j}^{\alpha \beta}\left(x_{0}, v\right)\right| \leq C \omega\left(|u-v|^{2}\right), \quad \forall x_{0} \in \Omega, \quad \forall u, v \in W_{l o c}^{1,2} .
$$

(H4) (Lower Order Items). We assume that $a_{i}^{\alpha}(x, u)$ and $b_{i}(x, u, \nabla u)$ are measurable for all $u \in W_{l o c}^{1,2}\left(\Omega, \mathbf{R}^{N}\right)$ with growth conditions

$$
\begin{gathered}
\left|a_{i}^{\alpha}(x, u)\right| \leq \mu_{1}\left(|u|^{\frac{\gamma}{2}}+f_{i}^{\alpha}(x)\right), \\
\left|b_{i}(x, u, \nabla u)\right| \leq \mu_{2}\left(|\nabla u|^{2\left(1-\frac{1}{\gamma}\right)}+|u|^{r-1}+g_{i}\right),
\end{gathered}
$$

where

$$
\begin{aligned}
& \gamma= \begin{cases}\frac{2 n}{n-2} & \text { if } n>2, \\
\text { any } \gamma>2 & \text { if } n=2,\end{cases} \\
& f_{i}^{\alpha} \in L^{p}(\Omega), \quad p>n, \quad g_{i} \in L^{q}(\Omega), \quad q>\frac{n}{2} ;
\end{aligned}
$$

for $\alpha=1,2, \cdots, n$ and $i=1,2, \cdots, N$.

\footnotetext{
${ }^{1}$ The continuous concave function $\omega(t)$ can be constructed by taking $\omega(t)=\inf \{\lambda(t)$ : $\lambda(t)$ concave and continuous with $\lambda(t) \geq \alpha(t)$ for any modulus of continuity $\alpha(t)$ which satisfies $\left.\left|A\left(x_{0}, u\right)-A\left(x_{0}, v\right)\right| \leq C \alpha\left(|u-v|^{2}\right)\right\}$.
} 
We summarize our main results by distinguishing the scalar case $N=1$ from the vectorial case $N>1$. That is, in the scalar case of $N=1$, we have:

Theorem 1. For $N=1$, let $u \in W_{\text {loc }}^{1,2}(\Omega, \mathbf{R})$ be a local weak solution of quasi-linear elliptic equation (1.1). Suppose that coefficients $A^{\alpha \beta}(x, u)$ satisfy assumptions (H1)-(H3), and the lower order items $a^{\alpha}(x, u)$ and $b(x, u, \nabla u)$ satisfy assumption (H4). Then we have $u \in C^{0, \kappa}(\Omega, \mathbf{R})$ for some $\kappa=\min \left\{1-\frac{n}{p}, 2-\frac{n}{q}\right\}$.

In the vectorial case of $N>1$, we have:

Theorem 2. For $N>1$, let $u \in W_{l o c}^{1,2}\left(\Omega, \mathbf{R}^{N}\right)$ be a local weak solution of quasi-linear elliptic system (1.1). Suppose that coefficient operators $A_{i j}^{\alpha \beta}(x, u)$ satisfy assumptions (H1)-(H3), and the lower order items $a_{i}^{\alpha}(x, u)$ and $b_{i}(x, u, \nabla u)$ satisfy assumption (H4). Then there exists an open subset $\Omega_{0} \subset \Omega$ with $\operatorname{dim}_{H}(\Omega \backslash$ $\left.\Omega_{0}\right) \leq n-2$ such that $u \in C^{0, \kappa}\left(\Omega_{0}, \mathbf{R}^{N}\right)$ for some $\kappa=\min \left\{1-\frac{n}{p}, 2-\frac{n}{q}\right\}$.

The rest of the paper is organized as follows. In Section 2, to make our paper sufficiently self-contained, we introduce several technical lemmas. In Section 3, we present our proofs for Theorems 1 and 2. Section 4 presents a brief conclusion.

\section{Preliminaries}

For our convenience, in the following context we use the notation

$$
A_{i j}^{\alpha \beta}\left(\cdot, u_{x_{0}, R}\right)_{x_{0}, R}=f_{B_{R}\left(x_{0}\right)} A_{i j}^{\alpha \beta}\left(y, u_{x_{0}, R}\right) d y .
$$

Let $v(x)$ be a weak solution to the Dirichlet problem:

$$
\left\{\begin{array}{l}
D_{\alpha}\left(\left(A_{i j}^{\alpha \beta}\left(\cdot, u_{x_{0}, R}\right)_{x_{0}, R}\right) D_{\beta} v^{j}\right)=0, \quad i=1,2, \cdots, N \quad \text { in } B_{R}\left(x_{0}\right) \\
v-u \in W_{0}^{1,2}\left(B_{R}\left(x_{0}\right), \mathbf{R}^{N}\right) .
\end{array}\right.
$$

In order to present proofs of our main results in a straightforward manner, here we introduce several technical lemmas:

Lemma 1. (Jensen's inequality [24]) Let $\omega: \mathbf{R} \rightarrow \mathbf{R}$ be a concave function. For any bounded open subset $U \subset \mathbf{R}^{n}$, suppose that $u: U \rightarrow \mathbf{R}$ is an integrable function. Then we have

$$
f_{U} \omega(u) d x \leq \omega\left(f_{U} u d x\right)
$$

Lemma 2. [4] Let $v(x) \in W^{1,2}\left(B_{R}, \mathbf{R}^{N}\right)$ be a weak solution of system (2.1). Then there exists a constant $C=C(\nu, L)$ such that for any $x_{0} \in \Omega$ and $0<\rho<$ $R \leq \operatorname{dist}\left(x_{0}, \partial \Omega\right)$, there holds

$$
\int_{B_{\rho}\left(x_{0}\right)}|D v|^{2} d x \leq C\left(\frac{\rho}{R}\right)^{n} \int_{B_{R}\left(x_{0}\right)}|D v|^{2} d x .
$$

Lemma 3. (Iteration Lemma [2, 10]) For any fixed $x_{0} \in \Omega$, let $\Phi(\rho)$ be a nonnegative and nondecreasing function. Suppose that

$$
\Phi(\rho) \leq A\left[\left(\frac{\rho}{R}\right)^{a}+\epsilon\right] \Phi(R)+B R^{b}, \quad \text { for all } 0<\rho<R \leq R_{0}=\operatorname{dist}\left(x_{0}, \partial \Omega\right),
$$


where $A, B, a, b$ are nonnegative constants with $a>b$. Then there exist positive constants $\epsilon_{0}=\epsilon_{0}(A, a, b)$ and $C=C(A, a, b)$ such that if $\epsilon<\epsilon_{0}$, such that

$$
\Phi(\rho) \leq C\left[\left(\frac{\rho}{R}\right)^{b} \Phi(R)+B \rho^{b}\right], \quad \forall 0<\rho<R<R_{0} .
$$

Lemma 4. (Reverse Hölder Inequality [4]) Let $u \in W_{\text {loc }}^{1,2}\left(\Omega, \mathbf{R}^{N}\right)$ be a local weak solution of system (1.1) under assumptions of (H1)(H2) and (H4). Then $D u \in L_{l o c}^{r}\left(\Omega, \mathbf{R}^{N}\right)$ for some $r>2$. Moreover, there exist constants

$$
C=C\left(n, \nu, \mu_{1}, \mu_{2}, p, q, L\right)>0
$$

and $\bar{R}>0$, for each ball $B_{R}\left(x_{0}\right) \subset \Omega$ when $0<R<\bar{R}$, such that

$$
\begin{aligned}
& \left(f_{B_{\frac{R}{2}}}\left(|u|^{\gamma}+|D u|^{2}\right)^{\frac{r}{2}} d x\right)^{\frac{1}{r}} \leq C\left(f_{B_{R}}\left(|u|^{\gamma}+|D u|^{2}\right) d x\right)^{\frac{1}{2}} \\
+ & C\left(f_{B_{R}} \sum_{\alpha, i}\left|f_{i}^{\alpha}(x)\right|^{r} d x\right)^{\frac{1}{r}}+C R\left(f_{B_{R}} \sum_{i}\left|g_{i}(x)\right|^{\frac{\gamma}{\gamma-1} \frac{r}{2}} d x\right)^{\frac{2}{r} \frac{\gamma-1}{\gamma}},
\end{aligned}
$$

which $\gamma$ is the same exponent as given in (1.4).

Since $\Phi_{p}(f)=\left(\frac{1}{\left|B_{R}\right|} \int_{B_{R}}|f(x)|^{p} d x\right)^{\frac{1}{p}}$ is non-decreasing in $p>0$ for any fixed $f(x)[1,24]$, from $(2.3)$ we have

$$
\begin{aligned}
\left(f_{B_{\frac{R}{2}}}|D u|^{r} d x\right)^{\frac{2}{r}} & \leq\left(f_{B_{\frac{R}{2}}}\left(|u|^{\gamma}+|D u|^{2}\right)^{\frac{r}{2}} d x\right)^{\frac{2}{r}} \\
& \leq C\left(f_{B_{R}}\left(|u|^{\gamma}+|D u|^{2}\right) d x\right)+C\left(f_{B_{R}} \sum_{\alpha, i}\left|f_{i}^{\alpha}(x)\right|^{p} d x\right)^{\frac{2}{p}} \\
& +C R^{2}\left(f_{B_{R}} \sum_{i}\left|g_{i}(x)\right|^{q} d x\right)^{\frac{2}{q}} .
\end{aligned}
$$

Lemma 5. (Dirichlet Growth Theorem [2]) Suppose that $u \in W^{1,2}\left(B_{R}\left(x_{0}\right), \mathbf{R}^{N}\right)$ satisfies the inequality:

$$
\int_{B_{R}}|\nabla u|^{2} d x \leq M^{2} R^{n-2+2 \kappa},
$$

for any $B_{R}\left(x_{0}\right) \subset \Omega$, where $M$ is a positive constant and $\kappa \in(0,1)$. Then we have $u \in C_{l o c}^{\kappa}\left(\Omega, \mathbf{R}^{N}\right)$, and for any $\Omega^{\prime} \subset \subset \Omega$ there holds

$$
\sup _{\Omega^{\prime}}|u|+\sup _{x, y \in \Omega^{\prime}, x \neq y} \frac{|f(x)-f(y)|}{|x-y|^{\kappa}} \leq C\left[M+\|u\|_{L^{2}(\Omega)}\right],
$$

where $C=C\left(n, \kappa, \Omega^{\prime}, \Omega\right)>0$.

Lemma 6. (Estimate of Hausdorff Dimension [25]) Let $\Omega$ be an open subset of $\mathbf{R}^{n}$ and $u \in L_{\text {loc }}(\Omega)$. For $0 \leq s<n$ we set

$$
E_{s}:=\left\{x \in \Omega: \lim \inf _{\rho \rightarrow 0} \rho^{-s} \int_{B_{\rho}(x)}|u| d y>0\right\} .
$$


Then we have the estimate

$$
H^{s}\left(E_{s}\right)=0
$$

\section{Proofs of Main Results}

For any fixed $x_{0} \in \Omega$ and $0<R<\frac{d}{2}$ with $d=\operatorname{dist}\left(x_{0}, \partial \Omega\right)$, we know that $B_{2 R}:=B_{2 R}\left(x_{0}\right) \subset \Omega$. Suppose that $v(x)$ is the solution of the Dirichlet problem (2.1), by Lemma 2 , for $0<\rho<R$, then we immediately obtain

$$
\int_{B_{\rho}}|D u|^{2} d x \leq C\left(\frac{\rho}{R}\right)^{n} \int_{B_{R}}|D u|^{2} d x+C \int_{B_{R}}|D(u-v)|^{2} d x
$$

Set $w=u-v$. The difference between (1.2) and (2.1) yields

$$
\begin{aligned}
& \int_{B_{R}} A_{i j}^{\alpha \beta}\left(\cdot, u_{x_{0}, R}\right)_{x_{0}, R} D_{\beta} w^{j} D_{\alpha} \phi^{i} d x=\int_{B_{R}}\left[-a_{i}^{\alpha}(x, u) D_{\alpha} \phi^{i}+b_{i}(x, u, \nabla u) \phi^{i}\right] d x \\
+ & \int_{B_{R}}\left(A_{i j}^{\alpha \beta}\left(\cdot, u_{x_{0}, R}\right)_{x_{0}, R}-A_{i j}^{\alpha \beta}(x, u)\right) D_{\beta} u^{j} D_{\alpha} \phi^{i} d x
\end{aligned}
$$

for any $\phi \in W_{0}^{1,2}\left(B_{R}, \mathbf{R}^{N}\right)$. Since $w=u-v \in W_{0}^{1,2}\left(B_{R}, \mathbf{R}^{N}\right)$ with $w=0$ on $\partial B_{R}\left(x_{0}\right)$, we may take it as a test function. Taking into account the ellipticity assumption (H1), from (3.1) and (3.2) we have

$$
\begin{aligned}
\nu \int_{B_{R}}|D w|^{2} d x & \leq \int_{B_{R}} A_{i j}^{\alpha \beta}\left(\cdot, u_{x_{0}, R}\right)_{x_{0}, R} D_{\beta} w^{j} D_{\alpha} w^{i} d x \\
& =\int_{B_{R}}\left[-a_{i}^{\alpha}(x, u) D_{\alpha} w^{i}+b_{i}(x, u, \nabla u) w^{i}\right] d x \\
& +\int_{B_{R}}\left[A_{i j}^{\alpha \beta}\left(\cdot, u_{x_{0}, R}\right)_{x_{0}, R}-A_{i j}^{\alpha \beta}\left(x, u_{x_{0}, R}\right)\right] D_{\beta} u^{j} D_{\alpha} w^{i} d x \\
& +\int_{B_{R}}\left[A_{i j}^{\alpha \beta}\left(x, u_{x_{0}, R}\right)-A_{i j}^{\alpha \beta}(x, u)\right] D_{\beta} u^{j} D_{\alpha} w^{i} d x
\end{aligned}
$$

According to Young's inequality and Sobolev's inequality for $w \in W_{0}^{1,2}\left(B_{R}\right)$, there is a constant $C$ such that

$$
\begin{aligned}
\int_{B_{R}}|D w|^{2} d x & \leq C \int_{B_{R}}\left|a_{i}^{\alpha}(x, u)\right|^{2} d x+C\left(\int_{B_{R}}\left|b_{i}(x, u, \nabla u)\right|^{\frac{2 n}{n+2}} d x\right)^{\frac{n+2}{n}} \\
& +C \int_{B_{R}}\left|A_{i j}^{\alpha \beta}\left(\cdot, u_{x_{0}, R}\right)_{x_{0}, R}-A_{i j}^{\alpha \beta}\left(x, u_{x_{0}, R}\right)\right|^{2}|D u|^{2} d x \\
& +C \int_{B_{R}}\left|A_{i j}^{\alpha \beta}\left(x, u_{x_{0}, R}\right)-A_{i j}^{\alpha \beta}(x, u)\right|^{2}|D u|^{2} d x \\
& \equiv C(\mathrm{I}+\mathrm{II}+\mathrm{III}+\mathrm{IV}),
\end{aligned}
$$

where $I=\int_{B_{R}}\left|a_{i}^{\alpha}(x, u)\right|^{2} d x, I I=\left(\int_{B_{R}}\left|b_{i}(x, u, \nabla u)\right|^{\frac{2 n}{n+2}} d x\right)^{\frac{n+2}{n}}, I I I=$ $\int_{B_{R}}\left|A_{i j}^{\alpha \beta}\left(\cdot, u_{x_{0}, R}\right)_{x_{0}, R}-A_{i j}^{\alpha \beta}\left(x, u_{x_{0}, R}\right)\right|^{2}|D u|^{2} d x$, and $I V=\int_{B_{R}}\left|A_{i j}^{\alpha \beta}\left(x, u_{x_{0}, R}\right)-A_{i j}^{\alpha \beta}(x, u)\right|^{2}|D u|^{2} d x$.

Now we are going to estimate I, II, III and IV, respectively. For estimates of I, II and III, both the scalar and vectorial cases can be discussed in the same way, 
but for the estimate of IV, we need to divide discussions into the scalar case and vectorial case separately. First, for the cases of I and II, using assumption (H4) we have

$$
\begin{aligned}
I & =\int_{B_{R}}\left|a_{i}^{\alpha}(x, u)\right|^{2} d x \leq 2 \mu_{1} \int_{B_{R}}\left(|u|^{r}+\sum_{\alpha, i}\left|f_{i}^{\alpha}(x)\right|^{2}\right) d x \\
& \leq C \int_{B_{R}}|u|^{r} d x+C R^{n-2+2 \kappa_{1}}\left(\int_{B_{R}} \sum_{\alpha, i}\left|f_{i}^{\alpha}(x)\right|^{p} d x\right)^{\frac{2}{p}}, \\
I I & =\left(\int_{B_{R}}\left|b_{i}(x, u, \nabla u)\right|^{\frac{2 n}{n+2}} d x\right)^{\frac{n+2}{n}}, \\
& \leq C\left(\int_{B_{R}}\left(|\nabla u|^{2}+|u|^{r}+\sum_{i}\left|g_{i}(x)\right|^{\frac{2 n}{n+2}}\right) d x\right)^{\frac{n+2}{n}}, \\
& \leq C\left(\int_{B_{R}}\left(|\nabla u|^{2}+|u|^{r}\right) d x\right)^{1+\frac{2}{n}}+C R^{n-2+2 \kappa_{2}}\left(\int_{B_{R}} \sum_{i}\left|g_{i}(x)\right|^{q}\right)^{\frac{2}{q}},
\end{aligned}
$$

where $\kappa_{1}=1-\frac{n}{p}$ and $\kappa_{2}=2-\frac{n}{q}$.

In order to estimate Part III, we make use of the VMO assumption (H2), inequality (1.3) as well as the reverse Hölder inequality (2.4), and have

$$
\begin{aligned}
\mathrm{III} & =\int_{B_{R}}\left|A_{i j}^{\alpha \beta}\left(\cdot, u_{x_{0}, R}\right)_{x_{0}, R}-A_{i j}^{\alpha \beta}\left(x, u_{x_{0}, R}\right)\right|^{2}|D u|^{2} d x, \\
& \leq\left(\int_{B_{R}}\left|A_{i j}^{\alpha \beta}\left(x, u_{x_{0}, R}\right)-A_{i j}^{\alpha \beta}\left(\cdot, u_{x_{0}, R}\right)_{x_{0}, R}\right|^{\frac{2 r}{r-2}} d x\right)^{\frac{r-2}{r}} \cdot\left(\int_{B_{R}}|D u|^{r} d x\right)^{\frac{2}{r}}, \\
& \leq C(r)\left(\omega_{n} R^{n}\right)^{\frac{r-2}{r}} \cdot M_{R}^{2}\left[A_{i j}^{\alpha \beta}\left(\cdot, u_{x_{0}, R}\right)\right] \cdot\left(\int_{B_{R}}|D u|^{r} d x\right)^{\frac{2}{r}}, \\
& \leq C M_{R}^{2}\left[A_{i j}^{\alpha \beta}\left(\cdot, u_{x_{0}, R}\right)\right] \cdot R^{n}\left(f_{B_{R}}|D u|^{r} d x\right)^{\frac{2}{r}}, \\
& \leq C R^{n} M_{R}^{2}\left[A_{i j}^{\alpha \beta}\left(\cdot, u_{x_{0}, R}\right)\right] \cdot\left[f_{B_{2 R}}\left(|u|^{\gamma}+|D u|^{2}\right) d x\right. \\
& \left.+\left(f_{B_{2 R}} \sum_{\alpha, i}\left|f_{i}^{\alpha}(x)\right|^{p} d x\right)^{\frac{2}{p}}+R^{2}\left(f_{B_{2 R}} \sum_{i}\left|g_{i}(x)\right|^{q} d x\right)^{\frac{2}{q}}\right] \\
& =C M_{R}^{2}\left[A_{i j}^{\alpha \beta}\left(\cdot, u_{x_{0}, R}\right)\right] \cdot \int_{B_{2 R}}\left(|u|^{\gamma}+|D u|^{2}\right) d x \\
& +C M_{R}^{2}\left[A_{i j}^{\alpha \beta}\left(\cdot, u_{x_{0}, R}\right)\right] \cdot\left[R^{n-\frac{2 n}{p}}\left(\int_{B_{2 R}} \sum_{\alpha, i}\left|f_{i}^{\alpha}(x)\right|^{p} d x\right)^{\frac{2}{p}}\right. \\
& +R^{n+2-\frac{2 n}{q}}\left(\int_{B_{2 R}} \sum_{i}\left|g_{i}(x)\right|^{q} d x\right)^{\frac{2}{q}} .
\end{aligned}
$$


Since $f_{i}^{\alpha}(x) \in L^{p}$ for $p>n$ and $f_{i}(x) \in L^{q}$ for $q>\frac{n}{2}$, we get

$$
\begin{aligned}
\text { III } & \leq C M_{R}^{2}\left[A_{i j}^{\alpha \beta}\left(\cdot, u_{x_{0}, R}\right)\right] \cdot\left[\int_{B_{2 R}}\left(|u|^{\gamma}+|D u|^{2}\right) d x\right. \\
& \left.+R^{n+2-2 \kappa_{1}} \sum_{\alpha, i}\left\|f_{i}^{\alpha}\right\|_{L^{p}}^{2}+R^{n+2-2 \kappa_{2}} \sum_{i}\left\|g_{i}\right\|_{L^{q}}^{2}\right],
\end{aligned}
$$

where $\kappa_{1}=1-\frac{n}{p}$ and $\kappa_{2}=2-\frac{n}{q}$.

On the other hand, if we set

$$
\gamma= \begin{cases}\frac{2 n}{n-2} & \text { if } n>2 \\ \text { any } \gamma>2 & \text { if } n=2,\end{cases}
$$

then there holds an inequality

$$
\begin{aligned}
\int_{B_{R}}|u|^{\gamma} d x & \leq C \int_{B_{R}}\left|u-u_{x_{0}, R}\right|^{\gamma}+C R^{n}\left|u_{x_{0}, R}\right|^{\gamma} \\
& \leq C\left(\int_{B_{R}}|\nabla u|^{2} d x\right)^{2 \gamma}+C R^{n}\left|u_{x_{0}, R}\right|^{\gamma} \\
& \leq C\left(\int_{B_{R}}|\nabla u|^{2} d x\right)^{\frac{\gamma}{n}} \cdot\left(\int_{B_{R}}\left(|u|^{\gamma}+|\nabla u|^{2}\right) d x\right)+C R^{n}\left|u_{x_{0}, R}\right|^{\gamma} .
\end{aligned}
$$

In order to estimate Part IV, we distinguish the proof in the scalar case from that in the vectorial case. Let us state a well-known result before starting to prove Theorem 1 in the scalar case. When $N=1$ we have a local Hölder continuity with some Hölder exponent $\sigma$ (undetermined) for the single equation (1.1):

LEMma 7. [1] Let $u(x) \in W^{1,2}\left(B_{R}, \mathbf{R}\right)$ be a local weak solution of equation (1.1), which satisfies assumptions (H1), (H2) and (H4). Then $u \in C^{\sigma}\left(B_{R}\right)$ for some $\sigma=\sigma\left(n, \nu, \mu_{1}, \mu_{2}, p, q\right)$ with $0<\sigma<1$. Moreover, there exists a positive constant $C=C\left(n, \nu, \mu_{1}, \mu_{2}, p, q\right)$ such that

$$
\|u\|_{C^{\sigma}\left(B_{R}\right)} \leq C\left(\|u\|_{L_{B_{R}}^{2}}+\sum_{\alpha}\left\|f^{\alpha}(x)\right\|_{L^{p}}+\|g(x)\|_{L^{q}}\right) .
$$

Now we are ready to prove Theorem 1.

Proof of Theorem 1. For the scalar case $N=1$, by assumption (H3) we have

$$
\begin{aligned}
\mathrm{IV} & =\int_{B_{R}}\left|A_{i j}^{\alpha \beta}\left(x, u_{x_{0}, R}\right)-A_{i j}^{\alpha \beta}(x, u)\right|^{2}|D u|^{2} d x \\
& \leq C \int_{B_{R}} \omega^{2}\left(\left|u-u_{x_{0}, R}\right|^{2}\right)|D u|^{2} d x \\
& \leq C \omega^{2}\left(C R^{2 \sigma}\right) \int_{B_{R}}\left(|u|^{\gamma}+|D u|^{2}\right) d x .
\end{aligned}
$$


When $N=1$, due to $u \in C^{\sigma}\left(B_{R}\right)$ from the conclusion of Lemma 7 , we know $\left|u_{x_{0}, R}\right| \leq M$. Substituting (3.4)-(3.8) into (3.3) and (3.1), we obtain

$$
\begin{aligned}
\int_{B_{\rho}}\left(|u|^{\gamma}+|D u|^{2}\right) d x & \leq C\left[\left(\frac{\rho}{R}\right)^{n}+\chi\left(x_{0}, R\right)\right] \int_{B_{2 R}}\left(|u|^{\gamma}+|D u|^{2}\right) d x+C R^{n} \\
& +C R^{n+2-2 \kappa_{1}} \sum_{\alpha, i}\left\|f_{i}^{\alpha}\right\|_{L^{p}\left(B_{2 R}\right)}^{2}+C R^{n+2-2 \kappa_{2}} \sum_{i}\left\|g_{i}\right\|_{L^{q}\left(B_{2 R}\right)}^{2} \\
& \leq C\left[\left(\frac{\rho}{R}\right)^{n}+\chi\left(x_{0}, R\right)\right] \int_{B_{2 R}}\left(|u|^{\gamma}+|D u|^{2}\right) d x+C R^{n+2-2 \kappa}
\end{aligned}
$$

where

$$
\begin{aligned}
\chi\left(x_{0}, R\right) & =\omega^{2}\left(C R^{2 \sigma}\right)+M_{R}^{2}\left[A_{i j}^{\alpha \beta}\left(\cdot, u_{x_{0}, R}\right)\right] \\
& +\left(\int_{B_{2 R}}|\nabla u|^{2} d x\right)^{\frac{\gamma}{n}}+\left(\int_{B_{2 R}}\left(|\nabla u|^{2}+|u|^{r}\right) d x\right)^{\frac{2}{n}} .
\end{aligned}
$$

Due to $u \in W_{l o c}^{1,2}(\Omega, \mathbf{R})$, we know that $\int_{B_{R}}|\nabla u|^{2} d x$ and $\int_{B_{R}}|u|^{r} d x$ are absolutely continuous in $R$. With the addition of the modulus of continuity $\omega^{2}\left(C R^{2 \sigma}\right)$ and the VMO condition of $M_{R}^{2}\left[A_{i j}^{\alpha \beta}\left(x_{0}, u_{x_{0}, R}\right)\right]$, then $\chi\left(x_{0}, R\right) \rightarrow 0$ if $R \rightarrow 0$. By virtue of Lemma 3, from (3.9) we have

$$
\int_{B_{R}}\left(|u|^{\gamma}+|D u|^{2}\right) d x \leq C R^{n+2-2 \kappa}
$$

for any $0<R<R_{0} \leq \operatorname{dist}\left(x_{0}, \partial \Omega\right)$, where $\kappa=\min \left\{1-\frac{n}{p}, 2-\frac{2 n}{q}\right\}$. Using Lemma 5 it shows that $u \in C^{\kappa}(\Omega, \mathbf{R})$. This completes the proof of Theorem 1 .

Next, we prove Theorem 2 .

Proof of Theorem 2. In the vectorial case $N>1$, we use the assumption (H3), the Hölder inequality, the reserve Hölder inequality (2.3) and the Jensen inequality (2.2) of concave functions in Lemma 1, to consider the estimate of Part 
IV, then we have

$$
\begin{aligned}
\mathrm{IV} & =\int_{B_{R}}\left|A_{i j}^{\alpha \beta}\left(x, u_{x_{0}, R}\right)-A_{i j}^{\alpha \beta}(x, u)\right|^{2}|D u|^{2} d x \\
& \leq C \int_{B_{R}} \omega^{2}\left(\left|u-u_{x_{0}, R}\right|^{2}\right)|D u|^{2} d x \\
& \leq C\left(\int_{B_{R}}\left[\omega\left(\left|u-u_{x_{0}, R}\right|^{2}\right)\right]^{\frac{2 r}{r-2}} d x\right)^{\frac{r-2}{r}} \cdot\left(\int_{B_{R}}|D u|^{r} d x\right)^{\frac{2}{r}}, \\
& \leq C R^{n}\left(f_{B_{R}} \omega\left(\left|u-u_{x_{0}, R}\right|^{2}\right) \cdot\left[\omega\left(\left|u-u_{x_{0}, R}\right|^{2}\right)\right]^{\frac{r+2}{r-2}} d x\right)^{\frac{r-2}{r}} \cdot\left(f_{B_{R}}|D u|^{r} d x\right)^{\frac{2}{r}}, \\
& \leq C R^{n}\left(f_{B_{R}} \omega\left(\left|u-u_{x_{0}, R}\right|^{2}\right) d x\right)^{\frac{r-2}{r}} \cdot\left(f_{B_{R}}|D u|^{r} d x\right)^{\frac{2}{r}}, \\
& \leq C R^{n} \omega^{\frac{r-2}{r}}\left[f_{B_{R}}\left|u-u_{x_{0}, R}\right|^{2} d x\right] \cdot\left[f_{B_{2 R}}\left(|u|^{\gamma}+|D u|^{2}\right) d x\right. \\
& \left.+\left(f_{B_{2 R}} \sum_{\alpha, i}\left|f_{i}^{\alpha}(x)\right|^{p} d x\right)^{\frac{2}{p}}+R^{2}\left(f_{B_{2 R}} \sum_{i}\left|g_{i}(x)\right|^{q} d x\right)^{\frac{2}{q}}\right] .
\end{aligned}
$$

Using Sobolev's inequality again yields

$$
\begin{aligned}
\mathrm{IV} & \leq C \omega^{\frac{r-2}{r}}\left(C R^{2-n} \int_{B_{R}}|D u|^{2} d x\right) \cdot\left[\int_{B_{2 R}}\left(|u|^{\gamma}+|D u|^{2}\right) d x\right. \\
& \left.+R^{n+2-2 \kappa_{1}} \sum_{\alpha, i}\left\|f_{i}^{\alpha}\right\|_{L^{p}}^{2}+R^{n+2-2 \kappa_{2}} \sum_{i}\left\|g_{i}\right\|_{L^{q}}^{2}\right] .
\end{aligned}
$$

where $\kappa_{1}=1-\frac{n}{p}$ and $\kappa_{2}=2-\frac{n}{q}$.

Combining (3.1), (3.3)-(3.6) with (3.10), we derive

$$
\begin{aligned}
\int_{B_{\rho}}\left(|u|^{\gamma}+|D u|^{2}\right) d x & \leq C\left[\left(\frac{\rho}{R}\right)^{n}+\vartheta\left(x_{0}, R\right)\right] \cdot \int_{B_{2 R}}\left(|u|^{\gamma}+|D u|^{2}\right) d x \\
& +C R^{n}\left|u_{x_{0}, R}\right|^{\gamma}+C R^{n+2-2 \kappa}
\end{aligned}
$$

for any $0<\rho<R$, where

$$
\begin{aligned}
\vartheta\left(x_{0}, R\right) & =\omega^{\frac{r-2}{r}}\left(C R^{2-n} \int_{B_{R}}|D u|^{2} d x\right)+M_{R}^{2}\left[A_{i j}^{\alpha \beta}\left(\cdot, u_{x_{0}, R}\right)\right] \\
& +\left(\int_{B_{2 R}}|\nabla u|^{2} d x\right)^{\frac{\gamma}{n}}+\left(\int_{B_{2 R}}\left(|\nabla u|^{2}+|u|^{r}\right) d x\right)^{\frac{2}{n}} .
\end{aligned}
$$

Since

$$
R^{n}\left|u_{x_{0}, R}\right|^{\gamma} \leq \int_{B_{R}}|u|^{\gamma} d x
$$


Inequality (3.11) becomes

$$
\begin{aligned}
\int_{B_{\rho}}\left(|u|^{\gamma}+|D u|^{2}\right) d x & \leq C\left[\left(\frac{\rho}{R}\right)^{n}+\vartheta\left(x_{0}, R\right)\right] \cdot \int_{B_{2 R}}\left(|u|^{\gamma}+|D u|^{2}\right) d x \\
& +C R^{n+2-2 \kappa}+C \int_{B_{2 R}}|u|^{\gamma} d x
\end{aligned}
$$

Here let us recall the following quantity, which is called an excess $E(\rho)$ defined by

$$
E(\rho)=\rho^{2-n} \int_{B_{\rho}\left(x_{0}\right)}|D u|^{2} d x
$$

In view of [26], there exists a positive $\epsilon_{0}$ such that if $E\left(R_{0}\right)<\epsilon_{0}$ for some $R_{0} \leq$ $\operatorname{dist}\left(x_{0}, \partial \Omega\right)$, then we have

$$
E(\rho) \leq C\left(\frac{\rho}{R}\right)^{2 \alpha} E(R), \quad \forall \rho<R<R_{0},
$$

for some $0<\alpha<1$. In the following analysis, we need a bootstrap argument. By the reverse Hölder inequality (2.3), we know that $u^{\gamma} \in L_{l o c}^{1+\varepsilon}$ for some constant $\varepsilon>0$. Due to the fact that

or

$$
\left(f_{B_{R}}|u|^{\gamma} d x\right) \leq\left(f_{B_{R}}|u|^{\gamma(1+\varepsilon)} d x\right)^{\frac{1}{1+\varepsilon}},
$$

$$
R^{-\frac{n \varepsilon}{1+\varepsilon}} \int_{B_{R}}|u|^{\gamma} d x \leq C\left\|u^{\gamma}\right\|_{L^{1+\varepsilon}\left(B_{R}\right)},
$$

we have $u \in L_{l o c}^{\gamma, \sigma}(\Omega)$ for $\sigma=\frac{n \varepsilon}{1+\varepsilon}$. By (3.12) with the previous assumption $E\left(R_{0}\right)<$ $\epsilon_{0}$, thus we have $|\nabla u| \in L_{l o c}^{2, \sigma}(\Omega)$. By virtue of Sobolev's inequality it follows that

$$
\int_{B_{R}}\left|u-u_{x_{0}, R}\right|^{\gamma} d x \leq C\left(\int_{B_{R}}|D u|^{2} d x\right)^{\frac{\gamma}{2}} .
$$

Accordingly, we have $u \in L^{\gamma, \frac{\gamma}{2} \sigma}$. On the basis of definition (1.4) for $\gamma$, we get $\frac{\gamma}{2}>1$. We repeat the same procedure, then after finite steps an improvement can be reached with $\sigma>n-2+2 \kappa$. By means of the same arguments as described in $[2,4,6$, $10,27]$, it follows that $u \in C^{\kappa}\left(\Omega_{0}\right)$ for an open subset $\Omega_{0}$ with $\kappa=\min \left\{1-\frac{n}{p}, 2-\frac{n}{q}\right\}$, where

$$
\Omega \backslash \Omega_{0}=\left\{x \in \Omega: \liminf _{\rho \rightarrow 0} \rho^{2-n} \int_{B_{\rho}}|D u|^{2} d x>0\right\} .
$$

By virtue of Lemma 6, we obtain

$$
\mathcal{H}^{n-2}\left(\Omega \backslash \Omega_{0}\right)=0
$$

Therefore, we complete the proof of Theorem 2 .

\section{Conclusion}

In this work, we are concerned with regularity and partial regularity of weak solutions for the general quasi-linear elliptic equations and systems of divergence form, which contains the lower order items satisfying controllable growth in the Euclidean space. When coefficient operators $A_{i j}^{\alpha \beta}(x, u)$ and the lower order items $a_{i}^{\alpha}(x, u), b_{i}(x, u, \nabla u)$ of the systems (1.1) satisfy the given four conditions, we obtain 
two theorems for the scalar case $N=1$ and the vectorial case $N>1$, respectively.

It is worthwhile to mention that one may consider the same problem by modifying assumption (H4) under the natural growth. We are planning to present some interesting results in a forthcoming paper somewhere else.

\section{Acknowledgments}

The authors would like to thank Professor Gui-Qiang Chen for his kind suggestions. Part of contents has been announced at the Mathematics Seminar of Beijing

Jiaotong University, June 29, 2007. This work is also partially supported by UTPA Faculty Research Council Grant 119100.

\section{References}

[1] D. Gilbarg and N.S. Trudinger, Elliptic Partial Differential Equations of Second Order (3rd edition), Spinger-Verlag, Berlin, 2001.

[2] Q. Han and F.H. Lin, Elliptic Partial Differential Equations, Amer. Math. Soc., Providence, Rhode Island, 1997.

[3] M. Giaquinta and G. Modica, Regularity results for some classes of higher order nonlinear elliptic systems, J. Reine Angew. Math., 311 (1979), 145-169.

[4] M. Giaquinta, Multiple integrals in the calculus of variations and nonlinear elliptic systems, in 'Ann. Math. Stud.', Princeton Univ. Press, Vol. 105, 1983.

[5] M. Giaquinta, Introduction to Regularity Theory for Nonlinear Elliptic Systems, Birkhäuser Verlag, New York, 1993

[6] J. Nečas, Introduction to the Theorey of Nonlinear Elliptic Equations, Teubner VerlagsgeSellschaft, Leipzig, 1983.

[7] E. De Giorgi, Sulla differenziabilitá e l'analiticitá della estremali degli integrali multipli regolari. Mem. Accad. Sci,Torino cl. Sci. Fis. Mat. Nat., 3 (1957), 25-43.

[8] J. Nash, Continuity of solutions of parabolic and elliptic equations, Amer. J. Math., 8 (1958), 931-954.

[9] O.A. Ladyzhenskaya and N.N. Ural'tseva, Linear and Quasilinear Elliptic Equations, Nauka, Moscow, 1973

[10] C.B. Morrey, Multiple Integrals in the Calculus of Variations, Springer Verlag, New York, 1966.

[11] C. Hu, R. Temam and M. Ziane, Regularity results for linear elliptic problems related to the primitive equations, Chinese Ann. Math. (Ser. B), 23 (2002), 277-292.

[12] C. Hu, R. Temam and M. Ziane, Regularity for linear elliptic problems related to the primitive equations, (in Frontiers in Mathematical Analysis and Numerical Methods), pp. 149-170, World Science Publication, River Edge, NJ, 2004.

[13] J. Wolf, Partial regularity of weak solutions to nonlinear elliptic systems satisfying a Dini condition, Z. Anal. Anwendungen, 20 (2001), 315-330.

[14] D. Sarason, Functions of vanishing mean oscillation, Trans. Amer. Math. Soc., 207 (1975), 391-405.

[15] A. Maugeri, D.K. Palagachev and L.G. Softova, Elliptic and Parabolic Equations with Discontinuous Coefficients, Wiley-vch Verlag, Berlin, 2000.

[16] A. Maugeri, D.K. Palagachev and C. Vitanza, Oblique derivative problem for uniformly elliptic operators with VMO coefficients and applications, C. R. Acad. Sci. Paris. T., 327 (1998), 53-58.

[17] D.K. Palagachev, Quasilinear elliptic equations with VMO coefficients, Trans. Amer. Math. Soc., 347 (1995), 2481-2493.

[18] J. Kinnunen and S.L. Zhou A local estimate for nonlinear equations with discontinuous coefficients, Comm. Part. Diff. Equs., 24 (1999), 2043-2068.

[19] Y.M. Chen, Regularity of solutions to elliptic equations with VMO coefficients, Acta Math. Sinica, 20 (2004), 1103-1118.

[20] F. Chiarenza, M. Frasca and P. Longo, $W^{2, p}$ solvability of the Dirichlet problem for nonlinear elliptic equations with VMO coefficients, Trans. Amer. Math. Soc., 336 (1993), 841-853. 
[21] G. Di Fazio, On Dirichlet problem in Morrey spaces, Diff. Integ. Equs., 6 (1993), 383-391.

[22] P. Di Gironimo, L. Esposito and L. Sgambati. A remark on $L^{2, \lambda}$ regularity for minimizers of quasilinear functionals, Manuscripta Math., 113 (2004), 143-151.

[23] E.M. Stein, Harmonic Analysis: Real-variable Methods, Orthogonality, and Oscillatory Integrals, Princeton Univ. Press, Princeton, NJ, 1993.

[24] W.P. Ziemer, Weakly Differentiable Functions, Springer-Verlag, New York, 1989.

[25] L.C. Evans and R.F. Gariepy, Measure Theory and Fine Properties of Functions, in 'Studies in Advanced Mathematics', CRC Press, 1992.

[26] E. Giusti, Metodi Diretti nel Calcolo delle Variazioni, Unione Matematica italiana, Officine Grafiche Tecnoprint, Bologna, 1994.

[27] L. Simon, Theorems on Regularity and Singularity of Energy Minimizing Maps, BirkhauserVerlag, Berlin, 1996.

Department of Mathematics, Beijing Jiaotong University, Beijing 100044, China

Department of Mathematics, University of Texas-Pan American, Edinburg, TX 78539, USA

E-mail address: zsfeng@utpa.edu 